\title{
Papel de la instrucción en la solución de problemas de correlación
}

\author{
María Puy Pérez Echeverría \\ Mario Carretero Rodríguez \\ Departamento de Psicología Básica, Social y Metodológica \\ Universidad Autónoma de Madrid

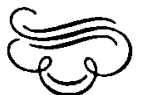 \\ Resumen
}

\begin{abstract}
Según varios autores, la instrucción sobre los conceptos de probabilidad y la experencia cotidiana en la resolución de problemas que implican esta noción mejora el rendimiento en la solución de problemas probabilísticos, incluso aunque se incluyan en áreas muy diferentes de las que se habia utilizado en el aprendizaje. Este artículo muestra una investigación sobre la forma en que solucionan problemas correlacionales licenciados universitarios en distintas especialidades que diferían tanto en su instrucción sobre estadística y probabilidad, como es su experiencia profesional con problemas probabilísticos. La correlación puede ser entendida como un concepto de probabilidad complejo y apenas existen investigaciones que estudien la influencia de la instrucción en problemas de este tipo concreto.
\end{abstract}

Nuestros sujetos fueron licenciados en Matemáticas, Psicología e Historia que estaban realizando cursos de postgrado y un grupo de licenciados en diferentes especialidades que estaban cursando un Master Internacional en Dirección de Empresas (MIDE). La tarea consistía en seis problemas correlacionales presentados mediante un cuestionario colectivo y que trataban de la relación entre las variaciones en el consumo de una bebida refrescante y una campaña de publicidad. Además de resolver los problemas los sujetos debían indicar la estrategia que creían baber utilizado para solucionarlos y debian escoger la mejor estrategia de solución entre un conjunto que se les presentaba, independientemente de si coincidía o no con la utilizada por ellos.

Nuestros resultados muestran que los matemáticos, seguidos por el grupo $M I D E$, resolvieron los problemas de forma más adecuada que los otros dos grupos. Estos mismos resultados se encuentran al analizar la adecuación de los autoinformes. Sin embargo, en este caso el grupo de psicólogos tuvo un rendimiento superior al del grupo de bistoriadores. No babía diferencias significativas en cuanto al reconocimiento de la estrategia más adecuada. Parece, por tanto, que en nuestro país la instrucción formal, tal y como la reciben los matemáticos, sirve para generalizar este tipo de conocimiento, mejor que la instrucción más pragmática que recibian los estudiantes del MIDE.

\section{Abstract}

According to several authors, the instruction in resolving and the experience with probabilistic problems in a particular area improve performance in probabilistic problems in general. This paper shows research about the performance in correlational problems of subjects who have graduated in several fields.

We tested mathematicians, psychologists, historicians and students of a Masters in Management International (MIDE) in a paper and pencil task. The task that the subjects had to do was composed by six correlational problems about the relationship between an advertisement and the frequency of consumption of a specific soft drink. The subjects had to specify the direction of the relationship in each problem. Also they bad to specify the strategies that they used to integrate information and they bad to specify the strategies that the used to integrate information and they bad to choose the best strategy among a number of strategies.

Our findings show that mathematicians performance was better than the MIDEs' but MIDEs' Performance was better than the other two groups. Mathematicians and MIDEs' also recognized the strategies used by themselves better than the other groups. Nevertheless there were not significant differences among the groups choosing the best strategy. It seems that a formal insstruction like what the mathematicians have received has better consequences in the performance of probabilistic problems in our country. 


\section{INTRODUCCION}

Durante los últimos años se está dedicando una especial atención a la forma en que sujetos adultos resuelven los problemas de correlación. Este interés está motivado por la importancia que tiene este concepto para la adaptación al medio ambiente, ya que la manera en que percibimos las contingencias es un indicio de la forma en que controlamos o creemos controlar el medio ambiente. El cálculo correlacional es, desde el punto de vista normativo, uno de los métodos más idóneos para detectar y medir la contingencia entre dos acontecimientos o entre nuestra conducta y las respuestas del medio.

La mayor parte de los trabajos sobre razonamiento correlacional se han centrado en dos aspectos. Por un lado, gran parte del esfuerzo campo se ha dirigido hacia el análisis de las estrategias y reglas utilizadas por sujetos adultos en la solución de problemas correlacionales con variables dicotómicas. Por otro lado, se ha estudiado el papel que juegan las preconcepciones y las teorías previas en esta resolución. El objetivo de este artículo es analizar cómo influyen algunas variables en las reglas de cálculo utilizadas por sujetos adultos en la solución de este tipo de problemas.

Desde el punto de vista normativo, la solución de un problema correlacional con variables dicotómicas exige que se realicen de forma multiplicativa las cuatro casillas de una tabla de contingencia (véase Tabla 1). Esta tabla resume los cuatro tipos de relación que se pueden dar entre dos acontecimientos. $\mathrm{La}$ casilla $\mathrm{A}$ nos informa sobre la frecuencia de aquellos casos en que las dos variables están presentes (p. q). La casilla $B$ hace referencia al número de ocasiones en los que la primera variable está ausente y la segunda presente (no p. q), mientras que la casilla $C$ incluye las situaciones en que la primera varible está presente y la segunda ausente (p. no q). La última casilla (D) recoge aquellos casos en que ninguna de las dos variables está presente.

TABLA 1: Tabla de contingencia utilizada para representar la correlación

\begin{tabular}{c|c|c|l} 
& $\mathrm{P}$ & no $\mathrm{P}$ & \\
\hline $\mathrm{q}$ & $\mathrm{A}$ & $\mathrm{B}$ & $\mathrm{A}+\mathrm{B}$ \\
\hline no $\mathrm{q}$ & $\mathrm{C}$ & $\mathrm{D}$ & $\mathrm{C}+\mathrm{D}$ \\
\hline & $\mathrm{A}+\mathrm{C}$ & $\mathrm{B}+\mathrm{D}$ & $\mathrm{A}+\mathrm{B}+\mathrm{C}+\mathrm{D}$
\end{tabular}

Aunque la solución correcta desde el punto de vista normativo implica que se pongan en relación multiplicativamente estas cuatro casillas, las investigaciones realizadas hasta la fecha han encontrado que los sujetos utilizan distintas reglas de cálculo (para una revisión véase Carretero, Pérez Echeverría 
y Pozo, 1985; Pérez Echeverría, 1988; Shaklee, 1982). A partir de la investigación pionera de Inhelder y Piaget (1955), estos trabajos han encontrado resultados muy diversos que muestran tanto que los sujetos adultos utilizan sólo la casilla A o comparan aditivamente la casilla A con la casilla B (por ejemplo, Smedlund, 1963), como que normalmente se utilizan las cuatro casillas de forma aditiva o multiplicativa (por ejemplo, Shaklee y Mims, 1982) o se realiza una comparación entre las diferencias de probabilidades (por ejemplo, Dickinson y Shanks, 1985). La utilización de una estrategia u otra parece depender de numerosos factores, tales como la forma de presentación de la tarea, el tipo de variables implicadas, la presencia de fuertes teorías previas, etc... y de los métodos experimentales utilizados en cada investigación concreta (Croker, 1981; Pérez Echeverría, 1988; Shaklee, 1982).

Sin embargo, apenas se ha estudiado la influencia de otros factores como la instrucción o la experiencia previa. No obstante, se ha comparado la actuación de las personas sin una especial preparación matemática con la forma en que teóricamente actuarían expertos que pusieran en juego sus conocimientos estadísticos. Algunos experimentos han comparado también la actuación de personas ingenuas con la actuación de psicólogos dedicados a la investigación (Tversky y Kahneman, 1973) o a la práctica clínica (Chapman, 1967; Chapman y Chapman, 1967, 1969, 1971). Sin embargo, apenas ha habido trabajos que investigasen la forma en que actúan distintos profesionales al enfrentarse con tareas de correlación.

Los trabajos realizados por Nisbett y sus colaboradores (Fong, Krantz y Nisbett, 1986; Kunda y Nisbett, 1985; Lehman, Lepert y Nisbett, 1986) con tareas de probabilidad estrechamente relacionadas con problemas de correlación indican que la experiencia previa puede actuar en dos sentidos. Por un lado, la experiencia cotidiana con un determinado contenido influye en la facilidad de codificación de los acontecimientos en problemas probabilísticos y, por tanto, en el procesamiento de la información y en la elaboración de conclusiones (Holland, Holyoak, Nisbett y Thagard, 1986). Por otro lado, una experiencia profesional con problemas de tipo probabilístico influye en que se utilicen estrategias probabilísticas para resolver tareas científicas ajenas al campo profesional de la persona y tareas de la vida cotidiana (Lehman, Lepert y Nisbett, 1986).

Vemos que, por tanto, los escasos trabajos que han controlado la experiencia previa de los sujetos en tareas probabilísticas han encontrado resultados diferentes en función de esta variable. Estos trabajos no han utilizado tareas de correlación en sus experimentos. Sin embargo, dado el amplio campo de problemas probabilísticos que han tratado, algunos de ellos relacionados con los errores más frecuentes en estas tareas («Falacia del jugador», «ley de los pequeños números», «intuiciones sobre la regresión»...), pensamos que este fenómeno puede darse también en la solución de problemas de correlación.

Por otro lado, se ha visto que los sujetos adultos somos muy poco conscientes de las reglas que utilizamos para resolver problemas de correlación (Pérez Echeverría, 1988). Salvo en el caso de utilización de las estrategias más correctas, las personas creemos que utilizamos estrategias más adecuadas que las que en realidad ponemos en juego (Shaklee y Hall, 1983). Si la experiencia con problemas probabilísticos influye en que se utilicen reglas más correctas desde el punto de vista normativo, es posible que influya también en la 
conciencia de las reglas utilizadas y en el reconocimiento de las estrategias más útiles para resolver el problema.

En el experimento que vamos a exponer a continuación tratamos de analizar la influencia de la experiencia en las estrategias utilizadas por distintos grupos de profesionales para resolver problemas de correlación, así como en el reconocimiento de los métodos utilizados y de las reglas más adecuadas para resolverlos. Por este motivo las tareas se han pasado a licenciados en distintas especialidades que estaban realizando cursos de postgrado y que tenían distintos tipos de experiencia con problemas probabilísticos. Los sujetos del experimento eran licenciados en Matemáticas que estaban realizando el CAP ( Curso de Aptitud Pedagógica) y cursos de Doctorado, licenciados en Ciencias Económicas, Empresariales o Derecho que estaban cursando un Master en Gerencia de Empresas (MIDE), licenciados en Geografía e Historia que estaban cursando el CAP y licenciados en Psicología que realizaban su especialización en Psicología Pedagógica.

Esperabamos que los estudiantes del MIDE y los licenciados en Matemáticas tuviesen un rendimiento superior a los otros dos grupos. Parte de la instrucción en el Master de Gerencia de Empresas se concentra en la toma de decisiones en situación de incertidumbre, utilizando para ello datos correlacionados. Además, como veremos más adelante, el contenido de los problemas propuestos a los sujetos estaba relacionado con el mundo empresarial. Por otro lado, los licenciado en matemáticas han recibido instrucción formal sobre estadística y solución de problemas matemáticos. Creíamos que estas características contribuirían a que la actuación de estos grupos sea superior a la actuación de los otros dos. Lehman, Lepert y Nisbett (1986) encontraron que la formación psicológica contribuía a un mejor rendimiento en tareas probabilísticas frente a otros profesionales. Sin embargo, nuestros experimentos con estudiantes españoles de Psicología (Carretero, Pérez Echeverría y Pozo, 1985) no indican este mejor rendimiento. Por este motivo creíamos que no habría diferencias a favor de los psicólogos en este experimento.

\section{Descripción de las pruebas}

Las tareas utilizadas consistían en seis problemas de correlación presentados mediante un cuadernillo y dos hojas de respuesta (véase Apéndice). Los sujetos debían consignar la posible existencia de una relación entre un anuncio publicitario presentado en seis cines y el consumo de una determinada bebida refrescante (Freshness) en los intermedios de la película que se estaba viendo en estos cines. Las instrucciones para realizar esta prueba estaban consignadas en los cuadernillos (véase Apéndice). Los datos se presentaban de forma integrada. Los seis problemas estaban diseñados de tal forma que por medio del conjunto de las respuestas se pudiera determinar cuál era la estrategia seguida por el sujeto para su resolución.

Los problemas utilizados y los resultados que se obtendrían en cada uno de ellos, según el tipo de estrategia utilizada, pueden verse en la Tabla 2. El signo « $t$ » indica que una estrategia determinada lleva al sujeto a predecir que el anuncio influye en que se beba más «Freshness» mientras que el signo «-» indica que el anuncio influye en que se beba menos «Freshness» y el signo «=» indica que no tiene influencia. El tipo de anuncio está representado por " $p$ " 
(nuevo anuncio) y por "no $p$ " (no nuevo anuncio). El refresco bebido esta representado por " $q$ " (Freshness) y por "no q" (no Freshness).

TABLA 2: Respuestas a cada problema en función de las estrategias utilizadas*

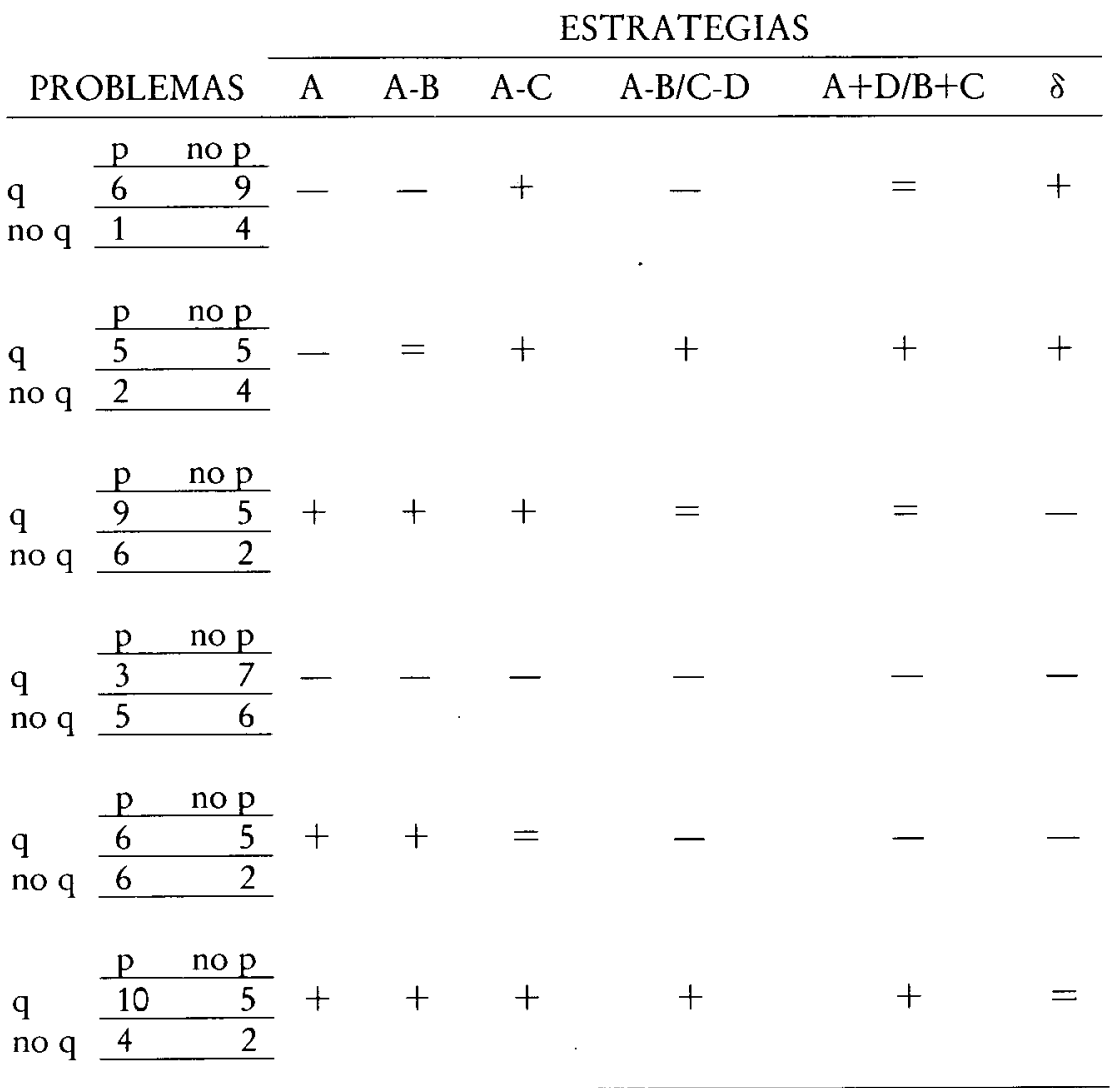

* Con el fin de nos cansar al lector con una descripción excesivamente larga de las estrategias consideradas a priori, hemos descrito sólo los métodos utilizados realmente por los sujetos. Además se consideraron todas las combinaciones posibles de dos casillas, todas las combinaciones aditivas posibles de cuatro casillas y la posibilidad de utilizar sólo la casilla B o la C.

Como se puede observar en la Tabla 2, hemos tenido en cuenta distintas estrategias posibles. En la tabla sólo se muestran aquellas que fueron utilizadas realmente por los sujetos. La regla más sencilla es la conocida como estrategia de la casilla $\mathrm{A}$. Consiste en comparar la frecuencia de esta casilla con las demás y en determinar si es más alta o más baja. Las dos siguientes estrategias se basan en la comparación entre dos casillas. Esta comparación se realiza mediante la sustracción del número de personas de las casillas $\mathrm{B}$ o $\mathrm{C}$ del número de personas de la casilla $\mathrm{A}([\mathrm{A}-\mathrm{B}]$ o $[\mathrm{A}-\mathrm{C}])$. Es decir, se comparan el número de personas que bebieron Freshness tras ver el nuevo anuncio (p. $q$ ) con el número de personas que tomaron Freshness sin ver el nuevo anuncio (no p. q), o con el número de personas que tras ver el anuncio no consumieron 
la mencionada bebida (p. no q). Las reglas 4 y 5 hacen referencia a la relación aditiva entre las cuatro casillas. La primera ([A-B]/[C-D]), consiste en determinar si la diferencias entre las personas que bebieron o no bebieron el producto son mayores tras ver el nuevo anuncio. Dicho de otra forma, consiste en comparar el resultado de ls sustracción entre las casillas A y B con el obtenido a partir de la resta entre las casillas $D$ y $C$. La quinta estrategia que hemos considerado es la comparación de la suma de diagonales $([\mathrm{A}+\mathrm{D}] /[\mathrm{B}+\mathrm{C}])$. Es decir, la comparación entre la suma de los casos que confirman la relación y la suma de los casos que falsan la relación. Para Inhelder y Piaget (1955) esta regla sería utilizada a partir del desarrollo de las operaciones formales. La última estrategia

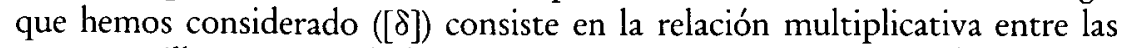
cuatro casillas. Aunque la hemos representado por medio de diferencias de probabilidades $(\delta)$, con otras estrategias (tantos por cientos, proporciones, etc...) que impliquen una relación multiplicativa se obtendrían los mismos resultados, acordes con lo predicho por las normas estadísticas.

Todas las reglas que acabamos de mencionar pueden agruparse ęn cuatro niveles en función del número de casillas utilizadas y el tipo de relación establecido entre ellas. El primer nivel está constituido por la estrategia [A]. Las soluciones que aporta esta estrategia sólo son correctas desde el punto de vista estadístico cuando esta casilla presenta una frecuencia muy superior a las otras tres casillas. El segundo nivel está formado por las reglas que relacionan aditivamente dos casillas ([A-B]y $[\mathrm{A}-\mathrm{C}])$. Estas estrategias llevan sólo a éxito cuando el resultado de estas diferencias es muy superior a las diferencias entre las casillas restantes. Las reglas que relacionan aditivamente las cuatro casillas forman el tercer nivel. Los resultados obtenidos con estas estrategias coinciden con los propuestos por la norma estadística cuando los valores marginales (suma de filas y/o suma de columnas) son iguales. La última estrategia constituye el cuarto nivel y, salvo errores de cálculo, lleva siempre a respuestas correctas.

Como hemos nombrado en el apartado anterior, no estábamos sólo interesados por las estrategias utilizadas para resolver problemas de correlación. También nos interesaba analizar el conocimiento que tienen sobre las estrategias que había utilizado y su capacidad para reconocer la estrategia óptima entre un grupo de estrategias. Una vez que se habían resuelto los seis problemas de correlación y que se había recogido su hoja de respuestas, se entregaba una segunda hoja en la que se definían siete estrategias diferentes (véase Apéndice). Los sujetos debían señalar aquella regla que se semejase más a la que creían haber utilizado y la regla que les pareciese más correcta. Estas hojas contaban con un espacio en blanco para describir la estrategia utilizada en el caso de que pensaran que ninguno de los métodos presentes era similar al empleado por ellos o se habían servido de varios métodos diferentes.

\section{Método}

\subsection{Sujetos}

La muestra estaba constituida por 130 sujetos ( 60 hombres y 70 mujeres) con una edad media de 26 años y 2 meses. La muestra estaba dividida en cuatro grupos en función de la licenciatura realizada y de los estudios de postgrado que se estaban cursando. 
El primer grupo de sujetos estaba constituido por 23 licenciados en Matemáticas que estaban cursando el CAP (Curso de Aptitud Pedagógica) en el ICE de la Universidad Autónoma de Madrid. Además, 16 de ellos estaban recibiendo cursos de doctorado y colaboraban con el Departamento de Matemáticas. El segundo grupo que tomó parte de este experimento, estaba constituido por 30 licenciados que estaban realizando un curso para obtener el Master Internacional en Dirección de Empresas (MIDE). De estos treinta sujetos, 14 eran licenciados en Ciencias Económicas o Empresariales, 7 habían obtenido su licenciatura en Derecho, 6 habían estudiado Ingeniería y los tres restantes habían obtenido distintos tipos de licenciatura. El tercer grupo estaba formado por 37 licenciados en Psicología que estaban cursando su especialización en Psicología Pedagógica. El cuarto grupo estaba formado por licenciados en Geografía e Historia que estaban realizando el Curso de Aptitud Pedagógica (CAP).

\subsection{Procedimiento}

Las tareas eran presentadas conjuntamente en un aula del centro donde los sujetos estaban cursando sus estudios de postgrado y se realizaban en presencia de un entrevistador y de alguno de sus profesores. Las instrucciones eran leídas en voz alta y se aclaraban las dudas a partir del ejemplo. No existía límite temporal para la realización de la tarea. Los sujetos contaban con lápiz y papel adicional para realizar lós cálculos o anotaciones que les fueran necesarios. Este material se recogía junto con las hojas de respuesta. Una vez finalizada esta primera parte, se repartía la segunda hoja de respuestas. Las instrucciones que contenía esta hoja también eran leídas en voz alta.

\section{Resultados}

\subsection{Número de respuestas correctas}

Como se ha podido ver antes, cada posible estrategia da lugar a un número de aciertos diferente en cada tarea. El primer análisis que hemos realizado ha sido el estudio del número de respuestas correctas alcanzado en función del grupo al que pertenecía el sujeto. Este análisis permite estudiar tanto la dificultad relativa de cada uno de los problemas como la influencia del tipo de instrucción recibido previamente.

El problema más fácil de resolver, si atendemos al porcentaje de respuestas correctas, fue el número 4 (82,32\% de respuestas correctas), ya que la obtención de un resultado correcto sólo se podía alcanzar por medio de la relación multiplicativa entre las cuatro casillas. El resto de los problemas fue resuelto por un porcentaje de sujetos que variaba entre el $50,8 \%$ y el $62,65 \%$.

Como puede observarse en la Tabla 3 , el grupo que obtuvo un mayor pcrcentaje de respuestas correctas fue el formado por los licenciados en matemáticas, seguido por el grupo de estudiantes del Master Internacional de Dirección de Empresas (MIDE). En los otros dos grupos el número de aciertos fue similar. Las diferencias entre los grupos resultaron significativas con una $p \leq 0.001(\mathrm{~F}=12.72)$. 
TABLA 3: Media y desviación típica del número de aciertos por persona

\begin{tabular}{lcc} 
& $\mu$ & $s$ \\
\hline Matemáticas & 5.3 & 1.4 \\
MIDE & 3.6 & 1.9 \\
Psicólogos & 2.6 & 1.9 \\
Geog. e Hist. & 2.7 & 1.6 \\
\hline
\end{tabular}

Por tanto, aparecen diferencias en este punto en función de la instrucción recibida. Los sujetos con una instrucción formal en matemáticas mostraron un rendimiento superior a los estudiantes entrenados en la toma de decisiones en el mundo empresarial y éstos a su vez tuvieron un porcentaje de éxitos superior a los otros dos grupos. La enseñanza de la estadística y la experincia con problemas probabilísticos del grupo de psicólogos parece tener un menor efecto que el aprendizaje en los otros dos campos mencionados.

\subsection{Estrategias utilizadas}

Sin embargo, la adecuación de la actuación de los sujetos en las tareas de correlación no depende sólo del número de respuestas correctas obtenidas en determinados problemas, sino también del método utilizado para obtener estas respuestas. En la Tabla 2 se mostraban una serie de estrategias que llevaban a distintas conclusiones en cada uno de los problemas. Los resultados obtenidos por cada sujeto se emparejaban con la estrategia que más se asemejaba a estos resultados. Para asignar un método a un individuo concreto era necesario que por lo menos cinco de sus respuestas fueran exactamente iguales a las predichas por esa estrategia concreta.

Como excepción a esta regla, se tenían en cuenta los cálculos consignados por los sujetos, aunque hubiese habido algún error de cálculo en las operaciones. Estas anotaciones fueron realizadas únicamente por sujetos que utilizaban las cuatro casillas tanto de forma aditiva como multiplicativa. Esta excepción es debida a que no nos interesaban los errores de cálculo que podían cometer los sujetos, sino las operaciones escogidas para integrar la información. De todas formas, hubo muy pocos errores, debido, seguramente, a que las cifras eran muy pequeñas.

El número de sujetos cuya estrategia no pudo ser incluida en ninguno de los grupos que habíamos considerado era de $10(7,7 \%)$. Por grupos, estos sujetos se distribuyen de la siguiente manera: Psicología $4(10,8 \%)$ y Geografía e Historia $6(10 \%)$. Como se puede observar, las respuestas que no se pudieron clasificar se dieron en los dos grupos que tuvieron un porcentaje mayor de errores. Ninguno de estos sujetos obtuvo un número de respuestas correctas superior a tres. Todos ellos fueron eliminados de los análisis que se expondrán a continuación. Por tanto, el número total de la muestra utilizada queda reducido a 120 sujetos.

Como veíamos antes, las estrategias mencionadas se pueden agrupar en cuatro niveles de complejidad creciente en función tanto del número de 
aciertos a que dan lugar como del número de casillas utilizados y del tipo de relación establecido entre las casillas. El nivel 1 está formado por los sujetos que utilizaron exlcusivamente la casilla A. El nivel 2 está compuesto por los sujetos que relacionaron de forma aditiva dos casillas (estrategias [A-B] y [AC]). El nivel 3 se forma con los estudiantes que relacionaron las cuatro casillas de forma aditiva (estrategias $[A-B] /[C-D]$ y $[A+D /[B+C]$. El nivel 4 estaba compuesto por los sujetos que utilizaron el método correcto desde el punto de vista normativo (estrategia [ $\delta]$. El método más utilizado fue la relación multiplicativa entre las cuatro casillas $(36,6 \%)$, es decir, la estrategia más cercana a la norma predicha. Las estrategias de los niveles 2 y 3 fueron empleadas por un porcentaje muy similar de sujetos $(22,5 \%$ y $25,8 \%$, respectivamente), mientras que sólo un $15 \%$ se centraron sólo en una casilla.

$\mathrm{Si}$ analizamos la actuación de los sujetos por grupos (véase Tabla 4), podemos observar que aparecen diferencias significativas medidas por la técnica de Chi cuadrado, tanto cuando se analizan las estrategias utilizadas por los distintos grupos como cuando se analizan estas diferencias tomadas dos a dos.

TABLA 4: Estrategias utilizadas para integrar la información

\begin{tabular}{lllll} 
& \multicolumn{1}{c}{1} & \multicolumn{1}{c}{2} & \multicolumn{1}{c}{3} & \multicolumn{1}{c}{4} \\
\hline Matemáticas & 0 & $3(13.04 \%)$ & 0 & $20(86.95 \%)$ \\
\hline MIDE & $4(13.3 \%)$ & $7(23.3 \%)$ & $7(23.3 \%)$ & $12(40 \%)$ \\
\hline Psicólogos & $8(24.2 \%)$ & $8(24.2 \%)$ & $11(33.3 \%)$ & $6(18.18 \%)$ \\
\hline Geog. e Hist. & $6(17.6 \%)$ & $9(26.5 \%)$ & $13(38.2 \%)$ & $6(17.6 \%)$ \\
\hline
\end{tabular}

Tomadas en conjunto las diferencias que acabamos de mencionar son significativas con el nivel de confianza de $1 \%\left(\mathrm{X}^{2}=31.4\right.$; g.1.=9). Como se puede observar, la actuación de los licenciados en Ciencias Exactas fue superior a la del resto de los grupos. Las diferencias entre este grupo y el resto de los grupos fueron significativas al nivel de confianza del $1 \%\left(\mathrm{X}^{2}{ }_{\mathrm{Mat} / \mathrm{MIDE}}=19.9\right.$; g. $1 .=3 ; \mathrm{X}^{2} \mathrm{Mat} / \mathrm{Psi}=28.15 ; \mathrm{g} .1 .=3 ; \mathrm{X}^{2} \mathrm{Mat} / \mathrm{G}$. H. $\left.=28.2 ; \mathrm{g} .1 .=3\right)$. También resultaron significativas con un nivel de confianza del $5 \%$ las diferencias entre las estrategias utilizadas por los estudiantes del MIDE y los otros dos grupos, cuando atendíamos a los dos niveles de respuesta superiores $\left(\mathrm{X}^{2} \mathrm{MIDE} / \mathrm{Psi}=4.01\right.$; g.1. $=1 ; \mathrm{X}^{2}$ MIDE/G. H. $=3.9 ;$ g.1.=1). Pero no resultaban significativas cuando se analizaban los cuatro niveles en conjunto. Tampoco resultaban significativas las diferencias entre los psicólogos y los licenciados en Geografía e Historia.

En resumen, existen diferencias significativas en función del tipo de instrucción recibida, tanto en lo referente al número de respuestas correctas en cada problema como en lo relativo a las estrategias empleadas para integrar la información. Estas últimas diferencias tienden a ser mayores en los dos niveles superiores. Si tuviéramos que ordenar los grupos en función de su actuación, el grupo con un rendimiento más alto estaría constituido por los 
licenciados en Matemáticas, seguido de los estudiantes del MIDE que, a su vez, parecen mostrar una actuación superior al de los licenciados en Psicología y al de los licenciados en Geografía e Historia.

\subsection{Estrategias que creen utilizar los sujetos y estrategias que consideran más adecuadas}

Como señalábamos en la introducción no sólo nos interesaba la forma en que se integra la información para resolver problemas de correlación, sino que también estábamos interesados en conocer la adecuación de los autoinformes sobre los métodos utilizados y el reconocimiento de las estrategias más adecuadas para solucionar este tipo de problemas.

Como en el análisis de las estrategias utilizadas, hemos agrupado tanto los métodos que los sujetos creen utilizar como los métodos que consideran mejores en cuatro niveles en función del número de casillas utilizado y la relación establecida entre esas casillas. Estos niveles son idénticos a los expuestos en el apartado anterior para clasificar las estrategias utilizadas. De esta clasificación se han eliminado los sujetos que no pudieron clasificarse en función de las estrategias utilizadas. El $55 \%$ de los sujetos informaban que habían utilizado estrategias correspondientes al nivel 4. Dicho con otras palabras, pensaban que habían establecido relaciones multiplicativas entre todas las frecuencias. Frente a este porcentaje, sólo el 7,5\% afirmaba haber utilizado una casilla exclusivamente. El número de sujetos que creía haber integrado dos casillas o cuatro casillas de forma aditiva alcanzaba el $24,6 \%$ y el $13,3 \%$, respectivamente.

En el análisis de los autoinformes de los distintos grupos no se encontraba ninguna diferencia significativa medida por la técnica Chi cuadrado al observar la actuación de los cuatro grupos en conjunto ni tomados dos a dos. Por tanto, podríamos decir que no existe ninguna diferencia entre los métodos que los sujetos manifiestan haber utilizado, sea cual sea el grupo al que pertenecen, y la forma en que hayan realizado la tarea. Sin embargo, como hemos visto antes, estas diferencias están presentes en las estrategias que han utilizado según nuestro análisis.

Un panorama semejante al que acabamos de describir surge cuando analizamos las estrategias que los sujetos han seleccionado como más adecuadas para relacionar los datos tras haber leído una serie de métodos. No existen diferencias entre los distintos grupos acerca de las estrategias escogidas como más adecuadas ni cuando se analizan conjuntamente ni cuando se analiza la actuación de cada grupo. El 66,66\% de los sujetos considera que las estrategias más adecuadas para resolver este tipo de problemas son las reglas de cálculo multiplicativas con los cuatro tipos de información. El 10,8\% considera más adeucadas las estrategias aditivas con las frecuencias de todas las casillas y el $17,5 \%$ piensa que las reglas más adecuadas son aquellas que relacionan entre sí sólo dos casillas. Por último, sólo el $5 \%$ piensa que basta examinar el número de sujetos que ven el nuevo anuncio e infieren el refresco ([A]).

No existe ninguna diferencia significativa entre los métodos que los sujetos consideran haber utilizado ni entre los métodos que consideran más adecuados para resolver los problemas de correlación. Sin embargo, si existen tales diferencias cuando se comparan con las estrategias utilizadas realmente como veremos en el siguiente apartado. 
3.4. Relación entre las estrategias utilizadas por los sujetos, autoinforme y estrategias que consideran más adecuadas

La relación entre los tres tipos de estrategias ha sido medida por medio de la Extensión de Mc Nemar de la prueba de Stuart para la Significación de los Cambios. Esta prueba mide los cambios que se producen en la actuación de cada sujeto en cada una de las variables, indicando el grado de significación de las diferencias en cada una de las categorías en que esta dividida la variable. Como en los casos anteriores, hemos utiliado como base para este análisis los cuatro niveles descritos anteriormente.

En el grupo de licenciados en Matemáticas que estaban cursando sus estudios de CAP y/o Doctorado, no existía ninguna diferencia entre los métodos que utilizaban según nuestro análisis y los que creían utilizar o consideraban más adecuados. De hecho el $100 \%$ de los sujetos informaba que había utilizado la misma estrategia que se desprendía de nuestros criterios. Estas reglas también coincidían totalmente con las que señalaban como más adecuadas.

Tampoco existían diferencias significativas entre los estudiantes del Master Internacional en Dirección de Empresas cuando analizabamos estos factores. Sin embargo, la coincidencia entre las reglas que habían utilizado para integrar la información según nuestro análisis y el autoinforme no era tan alta como en el grupo anterior. Aproximadamente el $70 \%$ de los sujetos señalaban que habían empleado las mismas reglas de cálculo que se desprendían de su actuación y consideraban estas reglas como las más adecuadas. En este porcentaje estaban incluidos todos los sujetos que relacionaron las cuatro casillas aditiva o multiplicativamente. El $30 \%$ restante consideraban que habían utilizado una estrategia mejor que la que se puede deducir del número y tipo de respuestas correctas obtenidas según nuestros criterios. Existía una coincidencia total entre los cálculos que manifestaban haber puesto en juego y las técnicas que consideraban más adecuadas.

La coincidencia entre el autoinforme, las estrategias empleadas y los métodos considerados mejores alcanzó el 31,25\% entre los psicólogos. En este porcentaje estaban incluidos todos los sujetos que utilizaron técnicas del nivel superior. Otro $12,5 \%$ consideraba que había utilizado las mismas reglas que se deducían a partir del tipo de respuestas que habían consignado, aunque no pensaban que estos métodos fueran los más adecuados tras haber leído otras alternativas. Prácticamente el $50 \%$ de este grupo consideraba que había utilizado estrategias de un nivel más alto que las que se extraían a partir de su actuación.

No existía ninguna diferencia significativa entre las estrategias utilizadas y las estrategias consignadas en el autoinforme. Sin embargo, sí aparecían diferencias significativas cuando se comparaba la actuación de los sujetos con las estrategias que creían más adecuadas para resolver este tipo de problemas. Estas diferencias se centraban en el cuarto nivel $\left(\mathrm{SE}_{4}^{2}=0.0153, \Delta_{4}=-0,7847\right.$ ) -0.0903 , g.1. $=3$;). Dicho en otras palabras, un número elevado de sujetos reconocían que el método más adecuado para resolver este tipo de problemas es la relación multiplicativa entre las cuatro casillas, aunque hubiesen empleado otro tipo de técnicas. Por tanto, se podría concluir que estos psicólogos tienen un conocimiento adecuado de las estrategias que utilizan, pero consideran que existen métodos más útiles para resolver los problemas de correlación. 
Este conocimiento que acabamos de describir no se manifiesta de la misma forma en el grupo compuesto por geógrafos e historiadores. En este caso existían diferencias significativas tanto entre las estrategias que se desprendían de su actuación y las reglas que manifestaban haber utilizado como entre los métodos utilizados y los que consideraba mejores $\left(\mathrm{SE}_{4}^{2}\right.$ ${ }_{\text {urilizar }}=0.0123, \Delta_{4}=-0,64 /-0.002, \mathrm{~g} .1 .=3, p=0.05 ;\left(\mathrm{SE}_{4}^{2}\right.$ urilizadas-mejor $=0.0137$, $\left.\Delta_{4}=-0.77 /-0.11, \mathrm{~g} .1 .=3, p=0.05\right)$. En otras palabras, un porcentaje significativo de los sujetos consideraba que había relacionado las cuatro casillas de forma multiplicativa, aunque el número y el tipo de respuestas indicase que habían utilizado reglas menos correctas. Además pensaban que el método multiplicativo era el más adecuado para solucionar estos problemas. No existían diferencias significativas entre las reglas que creían utilizar y las reglas que consideraban más adecuadas.

En resumen, podríamos decir que, excepto en este último grupo, había un conocimiento bastante adecuado de las normas utilizadas y que reconocían las normas más correctas para resolver este tipo de problemas. Cabe destacar que este conocimiento va disminuyendo en la medida en que las estrategias utilizadas se alejan más de las predichas por la norma. Es decir, conforme disminuye el rendimiento en la solución de los problemas, disminuye también la conciencia de las reglas que han utilizado y el conocimiento acerca de los métodos más adecuados para solucionar este tipo de tareas. Todos los sujetos que utilizaban las reglas más exactas, desde el punto de vista matemático, coincidían en aformar que sus métodos eran los más adecuados y tenían un conocimiento claro de la regla que habían utilizado. Sin embargo, los sujetos que no utilizaban estas reglas tendían a afirmar que habían utilizado métodos de un nivel superior.

\section{Conclusiones}

Al comentar los objetivos que nos habíamos propuesto con esta investigación, decíamos que nuestro principal objetivo era analizar la influencia de la instrucción y del tipo de problemas a los que se enfrentan los profesionales en la solución del problemas correlacionales.

Los resultados que hemos encontrado parecen indicar que la actuación de todos los grupos en estas tareas es bastante superior a la encontrada en otros trabajos realizados en este país con adultos (por ejemplo, Carretero, Pérez Echeverría y Pozo, 1985, véase Pérez Echeverría, 1988, para una revisión) y se asemejan a los encontrados por Shaklee y sus colaboradores en sus distintos trabajos con sujetos universitarios (Shaklee, 1983; Shaklee y Hall, 1983; Shaklee y Mims, 1981; Shaklee y Paszek, 1985; Shaklee y Tucker, 1980). De todas formas, en los trabajos realizados en nuestro país las pruebas fueron administradas a estudiantes universitarios, no a licenciados. Las diferencias entre este trabajo y los demás pueden reflejar precisamente las diferencias en la instrucción y en la experiencia con problemas de tipo probabilístico. La mayoría de los sujetos en cada grupo utilizó las cuatro casillas, bien de forma multiplicativa, bien de forma aditiva, para resolver estos problemas. Un porcentaje pequeño de licenciados se valieron sólo de la información precedente de dos casillas y un porcentaje aún menor se basó solamente en la casilla $\mathrm{A}$. 
Pero aunque la actuación de todos los grupos fue bastante adecuada, aparecían diferencias cuando se comparaban unos con otros. Sin lugar a dudas, el rendimiento de los licenciados en Matemáticas fue muy superior al rendimiento del resto de grupos, tanto en lo referente a las estrategias utilizadas como en lo referente a su conocimiento sobre las reglas que habían puesto en juego y a las normas que consideraban más adecuadas. Por tanto, en este caso se podría decir que la instrucción formal en problemas matemáticos favorece claramente el rendimiento en la solución de problemas de correlación.

Tras los matemáticos, el grupo que mostraba mejor actuación es el compuesto por los estudiantes del MIDE. Como comentábamos antes, este grupo tenía experiencia en la toma de decisiones en situación de incertidumbre con contenidos similares a los propuestos en la tarea, lo cual puede haber influido en que su actuación haya sido superior a la de los licenciados en Psicología y en Geografía e Historia. Este resultado parece estar en la línea de los encontrados por Nisbett y sus colaboradores (Fong, Krantz y Nisbett, 1986; Holand, Holyoak, Nisbett y Thagard, 1986; Kunda y Nisbett, 1985; Nisbett y Kunda, 1986). Según estos autores, la instrucción en estadística y probabilidad a través de la práctica influye en que se solucionen de forma más adecuada los problemas probabilísticos, incluso cuando están muy alejados de los contenidos que se han utilizado en el aprendizaje. Pero tampoco hay que olvidar que el contenido de las tareas era similar al de los problemas empleados en la enseñanza de este grupo. Este hecho ha podido contribuir a que se facilitase la codificación de las tareas y a que mejorase, por tanto, su actuación. Sin embargo, las diferencias con los matemáticos parecen mostrar que es más útil una formación general, más formal, en los conceptos y en los problemas matemáticos, que la formación más específica o más técnica que estaban recibiendo estos sujetos.

Por último, no existían diferencias entre la actuación de los psicólogos y los licenciados en Geografía e Historia. En principio se podría pensar que los psicólogos mostrarían un rendimiento superior debido tanto a su formación estadística como a su experiencia con problemas de carácter probabilístico, aunque esta hipótesis no está sustentada por los trabjos realizados en nuestro país, como veíamos en la introducción. De hecho, Lehman, Lepert y Nisbett (1986) encontraron que los psicólogos daban un mayor número de respuestas estadísticas a problemas cotidianos o a problemas profesionales que otros especialistas como médicos o abogados. Sin embargo, en el trabajo que acabamos de mencionar, las respuestas a los problemas consistían en explicaciones verbales sobre las causas de un determinado fenómeno. En ningún momento los sujetos tenían que resolver problemas numéricos. La experiencia profesional de los psicólogos con problemas probabilísticos suele referirse, salvo en ciertas ocasiones, a problemas de caso único. El trabajo de Lehman, Lepert y Nisbett estaba más centrado en este tipo de problemas. Es posible que las tareas planteadas por nosotros estuvieran más cerca de la experiencia profesional de los matemáticos o de los especialistas, en toma de decisiones empresariales, que de los psicólogos. Pero este tipo de argumentos no explicaría la ausencia de diferencias respecto al grupo de geógrafos e historiadores. Los psicólogos tienen un grado mayor de instrucción formal en estadística, incluyendo técnicas de solución de problemas probabilísticos y correlacionales, que el grupo anterior. También tienen teóricamente una mayor experiencia profesional en la solución de problemas probabilísticos. Quizá, a modo de hipótesis, las 
diferencias con los sujetos empleados por Lehman, Lepert y Nisbett (1986) residan en los diferentes métodos de instrucción que se sigue con los estudiantes de Psicología en España y en Estados Unidos. Sin embargo, cabe recordar también que otros trabajos con psicólogos americanos (Tversky y Kahneman, 1973; Campman y Chapman, 1969, 1971) encontraron que sus sujetos caían en errores similares a los de personas sin preparación científica al solucionar problemas en los que se debía tener en cuenta la Ley de los Grandes Números, el concepto de regresión a la media o que medían la influencia de las teorías previas en la resolución de los problemas de correlación.

Resumiendo, las diferencias en la instrucción y en la experiencia parecían favorecer al grupo de matemáticos y de estudiantes del Máster. Con ciertas dudas en lo relativo al grupo de psicólogos, podría afirmarse que la instrucción específica en problemas estadísticos y la experiencia con el contenido del problema favorecen el rendimiento en este tipo de tareas. De todas formas, dada la ausencia de trabajos sobre la solución de problemas de correlación que controlen la instrucción recibida por los sujetos, esta conclusión necesitaría ser contrastada con trabajos que incluyeran tanto otro tipo de profesionales como la actuación de personas adultas carentes de instrucción universitaria.

Otro de los objetivos de nuestra investigación fue analizar el conocimiento que tenían los sujetos de las reglas utilizadas y de las reglas que consideraban más adecuadas. De forma sorprendente hemos encontrado que todos los grupos mostraban un conocimiento bastante adecuado de las estrategias utilizadas, ya que no existían diferencias significativas entre su actuación y los métodos que consideraban haber utilizado. A pesar de ello, cuando se analizaba la actuación de los diferentes sujetos se veía que esta ausencia de diferencias estaba, en parte, motivada por los sujetos que utilizaban las reglas más correctas desde el punto de vista estadístico. Todos los sujetos que utilizaban estrategias multiplicativas con las cuatro casillas indicaban que se habían servido de esta regla. Conforme decrecía el uso de este método aumentaban las diferencias entre la estrategia utilizada y la estrategia que creían haber empleado. En la mayoría de los casos en que existían estas diferencias, los sujetos creían valerse de una regla más compleja que la que habían aplicado. Estos resultados están bastante en consonancia con los encontrados por Shaklee y Hall (1983). Pero la adecuación entre el autoinforme y las normas utilizadas, cuando no eran las correctas, fue superior en este estudio a la encontrada por Shaklee y Hall.

Sin embargo, hay que tener en cuenta que en este caso los sujetos tenían que reconocer las estrategias utilizadas dentro de un conjunto de reglas que se les daban ya redactadas. Los distintos licenciados no tenían que reconstruir los métodos que habían utilizado a partir de una introspección sobre su actuación. Sólo tenían que comparar las frases que aparecían en los cuadernillos con sus recuerdos sobre la forma en que habían trabajado. Dicho de otra forma, era una tarea de reconocimiento y no de producción en la que la carga en la memoria de trabajo era menor que en otros estudios. Además esta parte de la prueba se realizaba inmediatamente después de que hubieran solucionado los problemas. Estos hechos pueden haber influido en que los autoinformes hayan sido más adecuados que en otras investigaciones.

También los sujetos parecían reconocer de forma adecuada cuáles eran las reglas más correctas para resolver este tipo de problemas. Las diferencias entre su actuación y las normas que consideraban mejores parece indicar que 
reconocían las normas correctas independientemente de las que habían utilizado. De hecho, mientras que alrededor de un $37 \%$ de los sujetos empleaban las cuatro casillas multiplicativamente, casi un $68 \%$ reconocen esta estrategia como la más adecuada. Si a este porcentaje sumamos el del número de sujetos que considera más adecuada la utilización de las cuatro casillas de forma aditiva, nos encontramos que prácticamente el $80 \%$ de la muestra utilizada cree que es necesario tener en cuenta las cuatro casillas.

Los resultados obtenidos en el reconocimiento de las estrategias utilizadas y de las estrategias más adecuadas llevan a plantearse de nuevo las diferencias entre la producción y el reconocimiento en las tareas. Los sujetos parecen reconocer cuáles son los cálculos más apropiados para solucionar los problemas de correlación que se les plantean. Sin embargo, en algunos casos no los utilizan. Es posible que las características del problema (instrucciones, contenido, relaciones numéricas empleadas, etc.) influyan en que el sujeto no elicite estrategias que conoce al dejarse llevar más por las apariencias del problema que por su estructura. La influencia de estos factores se ha hecho patente en trabajos de comparación de proporciones y detección de probabilidades muy relacionados con el razonamiento correlacional (para una revisión de estos efectos, véase Pérez Echeverría, 1988, y Tourniaire y Pulos, 1985). De hecho, ésta parece ser una diferencia entre los expertos y no expertos al solucionar un problema (véase, por ejemplo, Chi, Feltovich y Glaser, 1981, y Pozo, 1987). Los matemáticos, expertos en la solución de problemas de este tipo, dejarían de lado estas características superficiales y actuarían partiendo de normas más generales que los otros grupos.

Las diferencias encontradas entre los distintos grupos también parece que la instrucción influye en estos dos últimos factores que estamos analizando. Como hemos dicho varias veces, la adecuación del autoinforme y el reconocimiento de las estrategias más adecuadas eran más apropiados en los sujetos que utilizan las normas más correctas. Por tanto, en general, los licenciados en Matemáticas y los estudiantes del MIDE tenían un rendimiento superior al de los otros dos grupos. Pero las diferencias entre los psicólogos y los geógrafos e historiadores parecen mostrar que la mayor instrucción de los primeros en temas relacionados con la estadística y la probabilidad facilita el reconocimiento tanto de la propia actuación como de los métodos necesarios para resolver estos problemas correctamente. Este hecho puede indicar que los psicólogos pueden percibir más claramente las contradicciones entre su actuación y la norma estadística $y$, por tanto, pueden resolver estas contradicciones más fácilmente.

Para finalizar, nos gustaría indicar que aunque en nuestro trabajo aparezcan diferencias en función de la instrucción, es necesario tener en cuenta que se han utilizado solamente licenciados universitarios y que el análisis no se ha realizado teniendo en cuenta todas y cada una de las diferencias en las enseñanza, sino tomándolas de forma global. Sería, por tanto, necesario analizar de forma más detallada estas diferencias así como comparar la actuación de sujetos con otros niveles de formación y experiencia con este tipo de problemas. 


\section{Referencias}

Carrletero, M. Pérez Echeverría, M. P. y Pozo, J. I.: El extraño caso del aceite de colza y la solución de problemas de correlación. Revista de Psicología General y Aplicada, 1985b, 40 (4), 703-725.

Crocker, J.: Judgment of covariation by social perceivers. Psychological Bulletin, 1981, 90 (2), 272-292.

Crocker, J..: Biased questions in judgment of covariation studies. Personality and Social Psychology Bulletin, 1982, 8, 214-220.

Chapman, L. J.: Illusory correlation in observational report.Journal of Verbal Learning and Verbal Behavior, 1967, 6, 151-155.

Chapman, L. J. y Chapman, J. P.: Genesis of popular but erroneous psychodiagnostic observations. Journal of Abnormal Psychology, 1967, 73, 193-104.

Chamman, L. J. y Chapman, J. P.: Illusory correlation as an obstacle to the use of valid psychodiagnostic signs. Journal of Abnormal Psychology, 1969, 74, 271-280.

Chapman, L. J. y Chapman, J. P.: Test results are what you think they are. Psychology Today, 1971 (November), 18-22, 106-110. También en D. Kahneman, P. Slovvic y A. Tversky (1982).

Chapman, R. P.: The development of children's understanding of prediction. Child Development, $1975,46,141-148$.

ChI, M. T. H.; Feltocich, P. J. y Glaser, R.: Categorization and representations of physics problems by experts and novices. Cognitive Science, 1981, S, 121-152.

Dickinson, A. y Shanks, D.: Animal conditioning and human causality judgment. En L. G. Nilsson y T. Archer (Eds.). Perspectives on Learning and Memory. Hillsdale, Nueva Jersey, LEA, 1985.

Fong, G., Krantz, D. H. y Nisbi:RTT, R.: The effects of statistical training in thinking about everyday problems. Cognitive Psychology, 1986, 18, 253-292.

Garcia Madruga, J. A. y Carretero, M.: Estrategias en el razonamiento humano. En $\mathrm{H}$. Peraita (coord.). Psicología cognitiva y ciencia cognitiva. Madrid. UNED, 1987.

Holland, J. H.; Holyoak, K. J.; Nisbltt, R. E. y Thagard, P. R.: Induction. Processes of inference, learning and discovery. Cambridge, Mass. MIT Press, 1986.

Inhlidir, B. y PAGet, J.: De la logique de l'enfant à la logique de l'adolescent. Essai sur la construction des structures operatoires formelles. París. PUF, 1955. Trad. castellama de M. T. Cevasco: De la lógica del niño a la lógica del adolescente. Buenos Aires. Paidós, 1972.

Kunda, Z. y NisBeTt, R.: The psychometrics of everyday life. Cognitive psychology, 1986, 18, 195-224.

Li:HMAN, D.; LEMPERT, R. y NISBETT, R. E.: "The effects of graduate education on reasoning". Manuscrito inédito. Universidad de Michigan, 1986.

NisbetT, R. y Kunda, Z.: Perception of social distribution. Journal of Personality and Social Psychology, 1985, 48, 297-311.

Pérliz Echeverria, M. P.: «Razonamiento probabilístico y correlacional: influencia de teorías previas y de datos». Tesis doctoral inédita. Departamento de Psicología Básica, Social y Metodológica. Madrid. Universidad Autónoma, 1988.

Pozo, J. I.: Aprendizaje de la ciencia y pensamiento causal. Madrid. Visor. Aprendizaje, 1987.

SHAKLEE, H.: Bounded rationality and cognitive development: upper limits on growth? Cognitive psychology, 1979, 11, 327-345.

SHAKLEE, H.: Human covariation judgment: accuracy and strategy. Learning and Motivation, $1983,14,433-448$.

ShakleE, H. y Hall, L.: Methods of assessing strategies for judging covariation between events. Journal of Educational Psychology, 1983, 75, 583-594.

ShaKLEE, H. y Mims, M.: Development of rule use in judgments of covariation between events. Child Development, 1981, 52, 317-325.

ShakleE, H. y PASzeck, D.: Covariation judgment: sistematic rule use in middle childhood. Child Development, 1985, 56, 1229-1240.

ShAKLEE, H. y TuCKer, D.: A rule analysis of judgments of covariation between events. Memory and Cognition, 1980, 8, 459-467.

Smbidiund, J.: The concept of correlation in adults. Scandinavian Journal of Psychology, 1963, 4, 165-174.

TVIRSKY, A. y KaHNEMAN, D.: Availability: a heuristic for judging frequency and probability. Cognitive Psychology, 1973, S, 207-232. (También en Kahneman, D., Slovic, p. y Tversky, A. (Eds.). Judgments under uncertainty: beuristics and biases. Nueva York. Cambridge University Press, 1982. 


\section{APENDICE: «FRESHNESS»}

En este cuadernillo vas a encontrar una serie de problemas sobre la relación entre la publicidad y la cantidad de bebida de una marca determinada que se consume en Europa.

Atiende a las explicaciones del profesor que te irá indicando lo que tienes que hacer. Al mismo tiempo debes ir leyendo las instrucciones. Si no entiendes algo, levanta la mano y él te lo explicara.

\section{NO ESCRIBAS NADA EN ESTE CUADERNILLO TUS RESPUESTAS DEBES PONERLAS EN LA HOJA DE RESPUESTAS}

"Freshness» es una importante empresa especializada en bel idas refrescantes con sabor a fruta. Sus bebidas son muy conocidas en toda Eı ropa.

Desde hace pocos meses, otras importantes empresas de este ramo están alcanzando una gran campaña publicitaria. Los encargados de "Freshness» están muy preocupados por esta campaña y, en consecuencia, están replanteándose su propia publicidad. Entre otras medidas, están estudiando la posibilidad de cambiar los anuncios publicitarios que se pasan en los cines. Hasta ahora «Freshness» insertaba en los pases de las películas unos anuncios que tenían gran aceptación entre el público. Parte de los publicistas de «Freshness» cree que un nuevo anuncio atraería a más público, sobre todo entre los jóvenes. Otra parte cree que la imagen de la empresa está muy consolidada y tiene un público fiel al que no le gustaría el cambio y, por lo tanto, el nuevo anuncio podría hacer que estas personas dejaran de tomar Freshness y bebiesen otros refrescantes. Un tercer grupo cree que el anuncio no iba a tener ninguna repercusión y que se bebería la misma cantidad de «Freshness» con un anuncio que con otro.

Antes de lanzar la nueva publicidad deciden hacer un estudio previo que mida su influencia. Para ello escogen seis cines importantes. Cada uno de ellos está situado en la capital de un país determinado. En todos estos cines están exponiendo la misma película y pasan el antiguo anuncio de «Freshness». Un sábado, en la sesión de tarde, cuentan el número de personas que beben «Freshness» y el número de personas que consumen otros refrescos en el intermedio de la película. Al sábado siguiente, en la misma sesión y durante la exhibición de la misma pelcula, pone el nuevo anuncio de «Freshness» en cada uno de estos cines y, luego, vuelven a contar el número de botellas de cada uno de los refrescos que se consumen.

Encontraron resultados diferentes en cada una de las ciudades del tipo de los que te vamos a presentar en el ejemplo siguiente. Todos los datos se refieren a los resultados encontrados en un solo cine. Los datos a y b corresponden a las personas que vieron la película el segundo sábado, cuando se introdujo el nuevo anuncio. Los datos $\mathrm{c}$ y $\mathrm{d}$ corresponden a las personas que vieron la película el primer sábado y por lo tanto no vieron el nuevo 
anuncio de Freshness, sólo el antiguo. En todas las sesiones se pasaban también anuncios de otras marcas de refrescos.

a) 10 personas vieron el nuevo anuncio y bebieron «Freshness».

b) 2 personas vieron el nuevo anuncio y no bebieron «Freshsness».

c) 2 personas no vieron el nuevo anuncio y bebieron «Freshness».

d) 2 personas no vieron el nuevo anuncio y no bebieron «Freshness».

Naturalmente, las personas que no vieron el nuevo anuncio habían visto el antiguo y las personas que no bebieron «Freshness" consumieron otras marcas de refrescos.

A partir de estos datos debes decidir si ocurre una de estas tres cosas:

1) El nuevo anuncio hace que se beba más «Freshness».

2) El nuevo anuncio hace que se beba menos "Freshness".

3) El nuevo anuncio no influye en que se beba más o menos "Freshness».

Elige tu respuesta e indicala en el lugar correspondiente de la $H O J A D E$ RESPUESTAS (donde dice EJEMPLO). Sólo tienes que tachar el número de la respuesta que creas correcta. Si te confundes rodea con un círculo la respuesta equivocada y responde de nuevo. Si necesitas hacer alguna anotación, hazla en la parte de atrás de la boja de respuestas.

En las páginas siguientes vas a encontrar seis problemas similares a éste. Cada uno de ellos te muestra los resultados de una ciudad distinta. Tienes que fijarte bien y responder en el lugar correspondiente de la HOJA DE RESPUESTAS, como en el ejemplo anterior.

NO EMPIECES A RESPONDER HASTA QUE SE TE INDIQUE. TOMATE EL TIEMPO QUE NECESITES PERO TRABAJA RAPIDO. 


\section{CIUDAD 1}

a) 6 personas vieron el nuevo anuncio y bebieron "Freshness".

b) 9 personas vieron el anuncio y no bebieron «Freshness».

c) 1 persona no vió el nuevo anuncio y bebió «Freshness».

d) 4 personas no vieron el nuevo anuncio y no bebieron «Freshness».

\section{CIUDAD 2}

a) 5 personas vieron el nuevo anuncio y bebieron "Freshness».

b) 5 personas vieron el anuncio y no bebieron "Freshness".

c) 2 personas no vieron el nuevo anuncio y bebieron "Freshness".

d) 4 personas no vieron el nuevo anuncio y no bebieron «Freshness».

\section{CIUDAD 3}

a) 9 personas vieron el nuevo anuncio y bebieron «Freshness».

b) 5 personas vieron el anuncio y no bebieron «Freshness».

c) 6 personas no vieron el nuevo anuncio y bebieron «Freshness».

d) 2 personas no vieron el nuevo anuncio y no bebieron «Freshness».

\section{CIUDAD 4}
a) 3 personas vieron el nuevo anuncio y bebieron "Freshness".
b) 7 personas vieron el anuncio y no bebieron «Freshness".
c) 5 personas no vieron el nuevo anuncio y bebieron "Freshness".
d) 6 personas no vieron el nuevo anuncio y no bebieron «Freshness».

\section{CIUDAD 5}
a) 6 personas vieron el nuevo anuncio y bebieron "Freshness».
b) 5 personas vieron el anuncio y no bebieron «Freshness».
c) 6 personas no vieron el nuevo anuncio y bebieron "Freshness".
d) 2 personas no vieron el nuevo anuncio y no bebieron «Freshness".

\section{CIUDAD 6}
a) 10 personas vieron el nuevo anuncio y bebieron «Freshness».
b) 5 personas vieron el anuncio y no bebieron "Freshness".
c) 4 personas no vieron el nuevo anuncio y bebieron "Freshness".
d) 2 personas no vieron el nuevo anuncio y no bebieron "Freshness". 
NOMBRE Y APELLIDOS:

FRESHNESS - HOJA DE RESPUESTAS

EJEMPLO:

1) El nuevo anuncio hace que se beba más «Freshness»..

2) El nuevo anuncio hace que se beba menos «Freshness».

3) El nuevo anuncio no influye en que se beba más o menos «Freshness».

\section{PRIMERA CIUDAD}

1) El nuevo anuncio hace que se beba más «Freshness».

2) El nuevo anuncio hace que se beba menos «Freshness».

3) El nuevo anuncio no influye en que se beba más o menos «Freshness».

\section{SEGUNDA CIUDAD}

1) El nuevo anuncio hace que se beba más «Freshness».

2) El nuevo anuncio hace que se beba menos «Freshness».

3) El nuevo anuncio no influye en que se beba más o menos «Freshness».

\section{TERCERA CIUDAD}

1) El nuevo anuncio hace que se bebá más «Freshness».

2) El nuevo anuncio hace que se beba menos «Freshness».

3) El nuevo anuncio no influye en que se beba más o menos «Freshness».

\section{CUARTA CIUDAD}

1) El nuevo anuncio hace que se beba más «Freshness».

2) El nuevo anuncio hace que se beba menos «Freshness».

3) El nuevo anuncio no influye en que se beba más o menos «Freshness».

\section{QUINTA CIUDAD}

1) El nuevo anuncio hace que se beba más «Freshness».

2) El nuevo anuncio hace que se beba menos "Freshness".

3) El nuevo anuncio no influye en que se beba más o menos «Freshness».

\section{SEXTA CIUDAD}

1) El nuevo anuncio hace que se beba más «Freshness».

2) El nuevo anuncio hace que se beba menos "Freshness".

3) El nuevo anuncio no influye en que se beba más o menos «Freshness". 
NOMBRE Y APELLIDOS:

EDAD: SEXO:

PROFESION:

ESTUDIOS ACTUALES:

\section{FRESHNESS}

Una vez que has resuelto los problemas anteriores queremos que nos digas la forma en que los has solucionado. A continuación encontrarás distintas frases que expresan distintos modos de buscar una solución a los ejercicios. Lee cada frase detenidamente y elige la que más se acerque al razonamiento que tú has realizado. Para ello tienes que tachar con una cruz el número que corresponde a esa frase en el lugar donde pone primera pregunta. Si te equivocas, rodea con un círculo la respuesta equivocada y responde de nuevo. En el caso en que creas que ninguna de las frases se corresponde con la forma en que has resuelto los problemas indícalo de la forma más clara y concisa posible donde pone otras. Si has seguido más de un método indícalo también donde pone otras, anotando el orden en que has seguido estos distintos métodos, bien con el número de la frase con que se corresponden, bien explicándolos.

Si después de haber leido estas frases crees que hay un método mejor para resolver los ejercicios que el que tú has elegido, tacha con una cruz el número de la frase que exprese, a tu juicio, la mejor forma de resolverlos en el lugar donde pone segunda respuesta. Si crees que han seguido el método más adecuado, vuelve a tachar el mismo número que en la primera pregunta. Lee cada frase con mucha atención ya que aunque algunas parecen semejantes, todas expresan métodos distintos.

1. Para saber como influye el nuevo anuncio hay que fijarse exclusivamente $s i$ el número de personas que lo vió y bebió «Freshness» en el intermedio es mayor que en cualquiera de los otros tres casos.

2. Para saber como influye el nuevo anuncio hay que fijarse exclusivamente $s i$ el número de personas que lo vió y bebió «Freshness» en el intermedio es mayor que el número de personas que vió el anuncio y tomaron otros refrescos.

3. Para saber como influye el nuevo anuncio hay que fijarse exclusivamente $s i$ el número de personas que lo vió y tomó «Freshness» es mayor que el número de personas que no vieron el nuevo anuncio y también tomaron «Freshness".

4. Para saber como influye el nuevo anuncio hay que fijarse exclusivamente $s i$ el número de personas que bebieron otros refrescos antes de ver el nuevo anuncio es mayor que el número de personas que bebieron otros refrescos después de verlo.

5. Para saber como influye el nuevo anuncio hay que fijarse exclusivamente $s i$ la división entre el número de personas que bebió «Freshness» antes y después de ver el nuevo anuncio es mayor que la división entre el número de personas que bebió otros refrescos antes y después de ver el nuevo anuncio.

6. Para saber como influye el nuevo anuncio hay que fijarse exclusivamente $s i$ el número de personas que bebió "Freshness» era mayor que el número de personas que bebió otros refrescos. 
7. Para saber como influye el nuevo anuncio hay que fijarse exclusivamente $s i$ la suma de las personas que vieron el nuevo anuncio y bebieron «Freshness" con las personas que ni lo vieron ni lo bebieron es mayor que la suma de los que bebieron «Freshness" sin ver el anuncio y de los que bebieron otros refrescos tras ver el anuncio.

$\begin{array}{llllllll}\text { PRIMERA PREGUNTA: } & 1 & 2 & 3 & 4 & 5 & 6 & 7\end{array}$

SEGUNDA PREGUNTA: $1 \quad 2 \quad \begin{array}{lllllll}1 & 2 & 3 & 4 & 5 & 6 & 7\end{array}$

OTRAS: 